Armen Edigarian (Kraków)

AgnieszKa Rygiel (Tarnów)

\title{
AN ELEMENTARY PROOF OF THE DALANG-MORTON-WILLINGER THEOREM
}

Abstract. L. C. G. Rogers has given an elementary proof of the fundamental theorem of asset pricing in the case of finite discrete time, due originally to Dalang, Morton and Willinger. The purpose of this paper is to give an even simpler proof of this important theorem without using the existence of regular conditional distribution, in contrast to Rogers' proof.

1. Introduction. Let $\left(\Omega, \mathfrak{F}_{T}, P\right)$ be a probability space and let $\mathfrak{F}_{0} \subset$ $\mathfrak{F}_{1} \subset \cdots \subset \mathfrak{F}_{T}$ be a finite filtration. At time $t \in\{0, \ldots, T\}$ the price vector of assets is a random variable $S_{t}: \Omega \rightarrow \mathbb{R}^{d}$. We define a portfolio strategy as a process $\theta_{t}: \Omega \rightarrow \mathbb{R}^{d}$. The interpretation is that $S_{t}^{i}$ is the discounted price of an asset and $\theta_{t}^{i}$ is the amount of the asset the investor holds in his portfolio. We suppose that the process $S$ is adapted to $\left(\mathfrak{F}_{t}\right)_{0 \leq t \leq T}\left(S_{t}\right.$ is $\mathfrak{F}_{t}$-measurable for all $\left.t=0, \ldots, T\right)$ and the process $\theta$ is predictable $\left(\theta_{t}\right.$ is $\mathfrak{F}_{t-1}$-measurable for all $\left.t=1, \ldots, T\right)$. We are interested in self-financing strategies only, i.e. portfolios without any exogenous infusion or withdrawal of money. The total gain (or loss) using such a strategy is then given by the $\operatorname{sum} \sum_{t=1}^{T}\left\langle\theta_{t}, S_{t}-S_{t-1}\right\rangle$.

An arbitrage opportunity is a portfolio strategy $\theta=\left(\theta_{0}, \theta_{1}, \ldots, \theta_{T}\right)$ with the properties

$$
\sum_{t=1}^{T}\left\langle\theta_{t}, S_{t}-S_{t-1}\right\rangle \geq 0 \quad \text { a.s., } \quad P\left(\sum_{t=1}^{T}\left\langle\theta_{t}, S_{t}-S_{t-1}\right\rangle>0\right)>0 .
$$

The result of Dalang, Morton and Willinger [1] is the following:

THEOREM 1. There does not exist an arbitrage opportunity if and only if there exists a probability $Q$ equivalent to $P$ such that $\left(S_{t}, \mathfrak{F}_{t}\right)_{t=0}^{T}$ is a

2000 Mathematics Subject Classification: 60G42, 91B28.

Key words and phrases: equivalent martingale measure, arbitrage. 
$Q$-martingale. In that case, it is possible to choose $Q$ in such a way that $d Q / d P$ is bounded.

If such a $Q$ exists, then $E_{Q}\left(\sum_{t=1}^{T}\left\langle\theta_{t}, S_{t}-S_{t-1}\right\rangle\right)=0$ for every predictable process $\theta=\left(\theta_{0}, \theta_{1}, \ldots, \theta_{T}\right)$. Since $\sum_{t=1}^{T}\left\langle\theta_{t}, S_{t}-S_{t-1}\right\rangle \geq 0$, we get $\sum_{t=1}^{T}\left\langle\theta_{t}, S_{t}-S_{t-1}\right\rangle=0$ almost surely. So, it is impossible to find an arbitrage opportunity.

It is well-known, and actually easy to show by an elementary induction argument, that for the proof of Theorem 1 it suffices to show the following (see e.g. [2]).

TheOREM 2. Let $(\Omega, \mathfrak{F}, P)$ be a probability space, let $X: \Omega \rightarrow \mathbb{R}^{d}$ be an $\mathfrak{F}$-measurable mapping, and let $\mathfrak{G}$ be a sub- $\sigma$-field of $\mathfrak{F}$. Then there does not exist a $\mathfrak{G}$-measurable mapping $\theta: \Omega \rightarrow \mathbb{R}^{d}$ such that

$$
\langle\theta, X\rangle \geq 0 \quad \text { a.s. and } P(\langle\theta, X\rangle>0)>0 .
$$

if and only if there exists a $\mathfrak{G}$-measurable mapping $\xi$ and a measure $Q$ such that $d Q=c e^{-\langle\xi, X\rangle-\|X\|^{2}-\|\xi\|^{2}} d P, X \in L^{1}(Q)$, and $E_{Q}(X \mid \mathfrak{G})=0$.

The aim of the paper is to give a truly elementary proof of Theorem 2 , and therefore, of Theorem 1. Our argument is a further simplification of L. C. G. Rogers' technique (see [3]). A simple, but conceptually more difficult proof is given by Yuri Kabanov and Christophe Stricker in [4]. However, their proof works well in the case of a model where the investor's decisions are based on partial information (see [5]). Probably, our strategy of proof cannot be successful in the restricted information case.

2. Proof of Theorem 2. Throughout this section we assume that $(\Omega, \mathfrak{F}, P)$ is a given probability space, $\mathfrak{G}$ is a sub- $\sigma$-field of $\mathfrak{F}$, and $X: \Omega \rightarrow \mathbb{R}^{d}$ is an $\mathfrak{F}$-measurable mapping. Fix a dense countable subset $\widetilde{D} \subset S^{d-1}$, where $S^{d-1}$ denotes the unit sphere in $\mathbb{R}^{d}$, and put $D=\left\{t x \in \mathbb{R}^{d}: x \in \widetilde{D}\right.$, $t \in \mathbb{Q}, t \geq 0\}$. Note that $D$ is a countable and dense subset of $\mathbb{R}^{d}$.

We first change to the probability measure $\widetilde{P} \sim P$ given by

$$
\frac{d \widetilde{P}}{d P}=c_{1} e^{-\|X\|^{2}},
$$

where $c_{1}^{-1}=E_{P}\left(e^{-\|X\|^{2}}\right)$. Then $E_{\widetilde{P}}\left(e^{\|X\|^{2}}\right)<\infty$ and the $\widetilde{P}$-integrability of $e^{-\langle a, X\rangle}$ is ensured for all $a \in \mathbb{R}^{d}$, because $e^{-\langle a, X\rangle} \leq e^{\|a\|^{2} / 4} \cdot e^{\|X\|^{2}}$.

Next we will show that there exists a $\mathfrak{G}$-measurable mapping with specific properties. This problem in various forms has been considered by L. C. G. Rogers (Proposition 2.4 in [3]) and F. Delbaen (Lemma 4.11 in [2]). 
LEMMA 3. There is a $\mathfrak{G}$-measurable matrix-valued mapping $R: \Omega \rightarrow$ $\mathbb{R}^{d \times d}$ such that

- $R R=R$ a.e. on $\Omega$;

- For any $\mathfrak{G}$-measurable mapping $\alpha: \Omega \rightarrow \mathbb{R}^{d}$ we have

$$
\langle\alpha, X\rangle=0 \text { a.s. } \Leftrightarrow R \alpha=\alpha \text { a.s. }
$$

In particular, $\langle R \alpha, X\rangle=0$ a.s.

Proof. For $i, j=1, \ldots, d$ we put $A_{i, j}(\omega)=X_{i}(\omega) X_{j}(\omega)$. Note that $A(\omega)=\left(A_{i, j}(\omega)\right)_{i, j=1}^{d}$ is a symmetric and non-negative definite $(d \times d)$ matrix. Next we define $B_{i, j}(\omega)=E_{\widetilde{P}}\left(A_{i, j} \mid \mathfrak{G}\right)(\omega)$ for $i, j=1, \ldots d$. Observe that for almost all $\omega$ the matrix $B(\omega)$ is also symmetric and non-negative definite. Indeed, $B_{i, j}=B_{j, i}$ almost surely and for each $q \in \mathbb{Q}^{d}$ we find a set $\Omega_{q}$ of probability one such that $q^{\prime} B(\omega) q=E_{\widetilde{P}}\left(q^{\prime} A q \mid \mathfrak{G}\right)(\omega) \geq 0$ for $\omega \in \Omega_{q}$. The countability of $\mathbb{Q}^{d}$ implies that there exists a set $\widehat{\Omega}$ such that $\widetilde{P}(\widehat{\Omega})=1$ and $q^{\prime} B(\omega) q \geq 0$ on $\widehat{\Omega}$ for every $q \in \mathbb{Q}^{d}$. Moreover, for every $x \in \mathbb{R}^{d}$ there exists a sequence $\left(q_{n}\right)_{n \in \mathbb{N}}$ of rational vectors such that $q_{n} \rightarrow x$. It follows that $x^{\prime} B x \geq 0$ for every $x \in \mathbb{R}^{d}$ on $\widehat{\Omega}$.

Fix an $\omega \in \Omega$. It is an elementary result from linear algebra that for $B(\omega)$ there exists an orthogonal matrix $Y(\omega)$ such that $Y(\omega) B(\omega) Y(\omega)^{-1}$ is a diagonal matrix. In other words, $B(\omega) \equiv Y^{-1}(\omega) \widetilde{B}(\omega) Y(\omega)$, where $\widetilde{B}(\omega)=$ $\operatorname{diag}\left(\lambda_{1}(\omega), \ldots, \lambda_{d}(\omega)\right)$ and $\lambda_{1}(\omega) \geq \cdots \geq \lambda_{d}(\omega) \geq 0$. Define

$$
R(\omega):=\lim _{t \rightarrow+\infty} e^{-t B(\omega)} \text {. }
$$

The $\mathfrak{G}$-measurability of $B$ implies that $R$ is also $\mathfrak{G}$-measurable. If for some $\omega \in \Omega$ the rank of $B(\omega)$ is $k$ and $\lambda_{i}(\omega) \neq 0$ for $i=1, \ldots, k$ then

$$
R(\omega)=Y(\omega)^{-1} \operatorname{diag}(0, \ldots, 0,1, \ldots, 1) Y(\omega),
$$

with $k$ zeros on the diagonal. From the definition we see that $R(\omega) R(\omega)=$ $R(\omega)$ for a.a. $\omega \in \Omega$.

Let $\alpha: \Omega \rightarrow \mathbb{R}^{d}$ be $\mathfrak{G}$-measurable. Note that the following conditions are equivalent:

- $\langle\alpha, X\rangle=0$ a.e. on $\Omega$;

- $\alpha^{\prime} A \alpha=0$ a.e. on $\Omega$;

- $\alpha^{\prime} B \alpha=0$ a.e. on $\Omega$.

Now fix $\omega \in \Omega$ and assume that $B(\omega)=Y(\omega)^{-1} \widetilde{B}(\omega) Y(\omega)$ (this is the previously used decomposition of $B(\omega))$. Put $\widetilde{\alpha}=Y(\omega) \alpha(\omega)$. Then $\alpha^{\prime}(\omega) B(\omega) \alpha(\omega)$ $=0$ if and only if $\widetilde{\alpha}_{1}(\omega)=\cdots=\widetilde{\alpha}_{k}(\omega)=0$. In a similar way $R(\omega) \alpha(\omega)=$ $\alpha(\omega)$ if and only if $\widetilde{\alpha}_{1}(\omega)=\cdots=\widetilde{\alpha}_{k}(\omega)=0$. Hence, $\langle\alpha, X\rangle=0$ a.e. on $\Omega$ if and only if $R \alpha=\alpha$ a.e. on $\Omega$. In particular, $\langle R \alpha, X\rangle=0$ a.e. on $\Omega$. 
We have $X_{1}^{j_{1}} \ldots X_{d}^{j_{d}} \in L^{1}(\widetilde{P})$ for any $j_{1}, \ldots, j_{d} \geq 0$. So, the functions $Y_{j_{1}, \ldots, j_{d}}(\omega)=E_{\widetilde{P}}\left(X_{1}^{j_{1}} \ldots X_{d}^{j_{d}} \mid \mathfrak{G}\right)(\omega)$ are well defined. We put

$$
\varphi(x ; \omega)=\sum_{j_{1}, \ldots, j_{d} \geq 0} \frac{(-1)^{j_{1}+\cdots+j_{d}}}{j_{1} ! \ldots j_{d} !} Y_{j_{1} \ldots j_{d}}(\omega) x_{1}^{j_{1}} \ldots x_{d}^{j_{d}} .
$$

We see that $\varphi(0 ; \omega)=1$ for a.a. $\omega \in \Omega$. Our aim is to show that $\varphi(\cdot ; \omega)$ is a real-analytic function for a.a. $\omega \in \Omega$. It suffices to show that the series in (4) is convergent for all $x \in D$ and for a.a. $\omega \in \Omega$. Define

$$
\varphi_{N}(x ; \omega)=\sum_{j_{1}, \ldots, j_{d} \geq 0, j_{1}+\cdots+j_{d} \leq N} \frac{(-1)^{j_{1}+\cdots+j_{d}}}{j_{1} ! \ldots j_{d} !} Y_{j_{1} \ldots j_{d}}(\omega) x_{1}^{j_{1}} \ldots x_{d}^{j_{d}} .
$$

Note that $\varphi_{N} \rightarrow \varphi$ a.s. Moreover,

$$
\begin{aligned}
\left|\varphi_{N}(x ; \omega)\right| & \leq \sum_{j_{1}, \ldots, j_{d} \geq 0} \frac{\left|Y_{j_{1} \ldots j_{d}}(\omega)\right|}{j_{1} ! \ldots j_{d} !}\left|x_{1}\right|^{j_{1}} \ldots\left|x_{d}\right|^{j_{d}} \\
& \leq e^{\|x\|^{2} / 4} E_{P}\left(e^{\|X\|^{2}} \mid \mathfrak{G}\right)(\omega) .
\end{aligned}
$$

For a.a. $\omega \in \Omega$ and for $x \in D$ we have $\varphi(x ; \omega)=E_{P}\left(e^{-\langle x, X\rangle} \mid \mathfrak{G}\right)(\omega)$. Hence, $\varphi(\cdot ; \omega)$ is convex for a.a. $\omega \in \Omega$.

We define the $\mathfrak{G}$-measurable convex function $\psi: \mathbb{R}^{d} \times \Omega \rightarrow[0, \infty)$ by

$$
\psi(x ; \omega):=\varphi(x ; \omega)+\|R(\omega) x\|^{2} .
$$

We denote by $B$ the set of all $\omega \in \Omega$ such that there exists a sequence $x_{n} \in \mathbb{R}^{d}$ with $\left\|x_{n}\right\| \rightarrow \infty$ and $\psi\left(x_{n} ; \omega\right) \leq 1$. Note that for a.a. $\omega \in \Omega \backslash B$ there exists a unique $a^{*}(\omega)$ at which $\psi(\cdot ; \omega)$ attains its minimum (the uniqueness follows from the analyticity and convexity of $\psi$ ).

We need the following.

FACT. Let $f: \mathbb{R}^{d} \rightarrow[0, \infty)$ be a convex function. Then the following conditions are equivalent:

(1) there exists a sequence $x_{n} \in \mathbb{R}^{d}$ such that

$$
\left\|x_{n}\right\| \rightarrow \infty \quad \text { and } \quad \limsup _{n \rightarrow \infty} f\left(x_{n}\right) \leq f(0)
$$

(2) there exists a sequence $x_{n} \in \mathbb{R}^{d}$ such that

$$
\left\|x_{n}\right\| \rightarrow \infty \quad \text { and } \quad \limsup _{n \rightarrow \infty} f\left(x_{n}\right)<\infty ;
$$

(3) there exists a point $x \in S^{d-1}$ such that $f(t x) \leq f(0)$ for any $t \geq 0$.

The only non-trivial implication is $(2) \Rightarrow(3)$. Fix a sequence $x_{n} \in \mathbb{R}^{d}$ so that $\left\|x_{n}\right\| \rightarrow \infty$ and $\limsup f\left(x_{n}\right)<\infty$. Note that there exists a subsequence so that $x_{n} /\left\|x_{n}\right\|$ is convergent to $x^{*} \in S^{d-1}$. We may assume that 
the sequence $x_{n}$ itself is convergent. Fix $t \geq 0$. Then for $m \geq t$ we have

$$
f\left(t \frac{x_{m}}{\left\|x_{m}\right\|}\right) \leq \frac{t}{\left\|x_{m}\right\|} f\left(x_{m}\right)+\frac{\left\|x_{m}\right\|-t}{\left\|x_{m}\right\|} f(0) .
$$

Let $m \rightarrow \infty$ to get $f\left(t x^{*}\right) \leq f(0)$.

Put $D_{m}=\{x \in D:\|x\| \geq m\}, m \in \mathbb{N}$. From the above Fact we have $B=\bigcap_{m=1}^{\infty} \bigcup_{x \in D_{m}}\{\omega \in \Omega: \psi(x ; \omega)<2\}$, and therefore $B \in \mathfrak{G}$.

For $j=1, \ldots, d$ we define $B_{j+}$ (resp. $B_{j-}$ ) as the set of all $\omega \in \Omega$ such that there exists a sequence $x_{n} \in \mathbb{R}^{d}$ with $\left\|x_{n}\right\| \rightarrow \infty, \psi\left(x_{n} ; \omega\right) \leq 1$, and $\left(x_{n}\right)_{j} \geq \varepsilon$ (resp. $\left(x_{n}\right)_{j} \leq-\varepsilon$ ) for some $\varepsilon>0$. Here $\left(x_{n}\right)_{j}$ denotes the $j$ th coordinate of $x_{n}$. Similarly to $B$, one can see that $B_{j+}, B_{j-} \in \mathfrak{G}$. Moreover, $\bigcup_{j=1}^{d}\left(B_{j+} \cup B_{j-}\right)=B$.

Our aim is to show that $\widetilde{P}(B)=0$. So, to get a contradiction we assume that $\widetilde{P}\left(B_{1+}\right)>0$. Let us show that there exists a $\mathfrak{G}$-measurable function $\theta: \Omega \rightarrow \mathbb{R}^{d}$ such that $|\theta(\omega)|=1$ for a.a. $\omega \in B_{1+}, \theta(\omega)=0$ for a.a. $\omega \notin B_{1+}$, and $\psi(t \theta(\omega) ; \omega) \leq 1$ for any $t \geq 0$. For any fixed $\omega \in B_{1+}$ there exists a unique $\theta(\omega) \in S^{d-1}$ such that $(\theta(\omega))_{1}$ is maximal. Indeed, if $\theta^{\prime}(\omega), \theta^{\prime \prime}(\omega) \in S^{d-1}$ and $\left(\theta^{\prime}(\omega)\right)_{1}=\left(\theta^{\prime \prime}(\omega)\right)_{1}$ is maximal and less than 1 , then for $\theta(\omega)=\left(\theta^{\prime}(\omega)+\theta^{\prime \prime}(\omega)\right) /\left\|\theta^{\prime}(\omega)+\theta^{\prime \prime}(\omega)\right\| \in S^{d-1}$ we have $\psi(t \theta(\omega) ; \omega) \leq 1$ for any $t \geq 0$ and $(\theta(\omega))_{1}>\left(\theta^{\prime}(\omega)\right)_{1}$. A contradiction. So, we have the uniqueness. Note that the function $\theta(\omega)$ is $\mathfrak{G}$-measurable.

We claim that $\langle\theta, X\rangle \geq 0$ a.s. and $P(\langle\theta, X\rangle>0)>0$. First note that

$$
\psi(t \theta(\omega) ; \omega)=E_{\widetilde{P}}\left(e^{-t\langle\theta, X\rangle} \mid \mathfrak{G}\right)(\omega)+t^{2}\|R(\omega) \theta(\omega)\|^{2}
$$

for a.a. $\omega \in \Omega$ and any $t \in \mathbb{Q}, t \geq 0$. So, we have $R(\omega) \theta(\omega)=0$ a.s.

For each $n$ we consider the events

$$
C_{n}=\left\{\omega \in B_{1+}:\langle\theta(\omega), X(\omega)\rangle \leq-1 / n\right\}
$$

and $C=\bigcup_{n \in \mathbb{N}} C_{n}$. We have

$$
E_{\widetilde{P}}\left(e^{-t\langle\theta, X\rangle} \mid \mathfrak{G}\right)(\omega) \geq E_{\widetilde{P}}\left(e^{-t\langle\theta, X\rangle} \chi_{C_{n}} \mid \mathfrak{G}\right)(\omega) \geq e^{t / n} E_{\widetilde{P}}\left(\chi_{C_{n}} \mid \mathfrak{G}\right)(\omega)
$$

for a.a. $\omega \in B$. The conclusion is that $E_{\widetilde{P}}\left(\chi_{C_{n}} \mid \mathfrak{G}\right)(\omega)=0$ for a.a. $\omega \in B$ and for all $n \in \mathbb{N}$. By using the monotone convergence theorem for conditional expectation we also have $E_{\widetilde{P}}\left(\chi_{C} \mid \mathfrak{G}\right)(\omega)=0$ for a.a. $\omega \in B$. Then

$$
0=\int_{B} E_{\widetilde{P}}\left(\chi_{C} \mid \mathfrak{G}\right) d \widetilde{P}=\int_{B} \chi_{C} d \widetilde{P}=\widetilde{P}(C) .
$$

So, $\langle\theta(\omega), X(\omega)\rangle \geq 0$ for a.a. $\omega \in \Omega$. Note that $\widetilde{P}(\langle\theta, X\rangle>0)>0$. Indeed, if $\langle\theta, X\rangle=0$ a.e. on $\Omega$, then $R \theta=\theta$; but $R \theta=0$ and $P(B)>0$, a contradiction. But the existence of such a $\theta$ is also a contradiction. So, we have proved that $\widetilde{P}(B)=0$.

Therefore, the set $\left\{x \in \mathbb{R}^{d}: \psi(x ; \omega) \leq 1\right\}$ is compact and there exists a unique $a^{*} \equiv a^{*}(\omega)$ such that $\psi\left(a^{*} ; \omega\right) \leq \bar{\psi}(a ; \omega)$ for all $a \in \mathbb{R}^{d}$. For any open 
ball $V \subseteq \mathbb{R}^{d}$ we have

$$
\left\{a^{*} \in V\right\}=\bigcup_{q \in \mathbb{Q}^{d} \cap V} \bigcap_{p \in \mathbb{Q}^{d} \backslash V}\{\psi(\omega ; q)<\psi(\omega ; p)\} .
$$

Since $\psi$ is $\mathfrak{G}$-measurable, (8) implies that $a^{*}$ is also $\mathfrak{G}$-measurable.

Now we show that $R(\omega) a^{*}(\omega)=0$ for this minimising choice. Suppose not, and define $\widetilde{a}(\omega):=a^{*}(\omega)-\frac{1}{2} R(\omega) a^{*}(\omega)$. For a.a. $\omega \in \Omega$ we have

$$
\langle\widetilde{a}(\omega), X(\omega)\rangle=\left\langle a^{*}(\omega), X(\omega)\right\rangle \text { and } R(\omega) \widetilde{a}(\omega)=\frac{1}{2} R(\omega) a^{*}(\omega),
$$

and therefore,

$$
\begin{aligned}
\psi\left(\omega ; a^{*}\right) & =E_{\widetilde{P}}\left(e^{-\left\langle a^{*}, X\right\rangle} \mid \mathfrak{G}\right)(\omega)+\left\|R(\omega) a^{*}\right\|^{2} \\
& >E_{\widetilde{P}}\left(e^{-\langle\widetilde{a}, X\rangle} \mid \mathfrak{G}\right)(\omega)+\frac{1}{4}\left\|R(\omega) a^{*}\right\|^{2}=\psi(\omega ; \widetilde{a}),
\end{aligned}
$$

a contradiction.

We have $\frac{\partial \psi}{\partial x_{k}}\left(a^{*}(\omega) ; \omega\right)=\frac{\partial \varphi}{\partial x_{k}}\left(a^{*}(\omega) ; \omega\right)=0$ for any $k=1, \ldots, d$, and therefore,

$$
E_{\widetilde{P}}\left(X_{k} e^{-\left\langle a^{*}(\omega), X\right\rangle} \mid \mathfrak{G}\right)(\omega)=0 \quad \text { for a.a. } \omega \in \Omega \text { and } k=1, \ldots, d .
$$

Note that

$$
0=E_{\widetilde{P}}\left(X_{k} e^{-\left\langle a^{*}(\omega), X\right\rangle-\left\|a^{*}(\omega)\right\|^{2}} \mid \mathfrak{G}\right)(\omega)=E_{\widetilde{P}}\left(X_{k} e^{-\left\langle a^{*}, X\right\rangle-\left\|a^{*}\right\|^{2}} \mid \mathfrak{G}\right)(\omega)
$$

for a.a. $\omega \in \Omega$ and $k=1, \ldots, d$. Now it suffices to take $\xi=a^{*}$.

\section{References}

[1] R. C. Dalang, A. Morton and W. Willinger, Equivalent martingale measures and no-arbitrage in stochastic securities market models, Stoch. Stoch. Rep. 29 (1990), 185-201.

[2] F. Delbaen, The Dalang-Morton-Willinger theorem, preprint, 1999.

[3] L. C. G. Rogers, Equivalent martingale measure and no-arbitrage, Stoch. Stoch. Rep. 51 (1994), 41-49.

[4] Yu. M. Kabanov and C. Stricker, A teachers' note on no-arbitrage criteria, in: Séminaire de Probabilités XXXV, Lecture Notes in Math. 1755, Springer, 2001, 149-152.

[5] - - - The Dalang-Morton-Willinger theorem under delayed and restricted information, in: Séminaire de Probabilités XXXIX, Lecture Notes in Math. 1874, Springer, 2006, 209-213.

Institute of Mathematics

Jagiellonian University

Reymonta 4

30-059 Kraków, Poland

E-mail: Armen.Edigarian@im.uj.edu.pl
Higher Vocational School in Tarnów Mickiewicza 8 33-100 Tarnów, Poland E-mail: Agnieszka.Rygiel@im.uj.edu.pl

Received on 28.8.2007;

revised version on 20.11.2007 